\title{
EDITORIAL
}

\section{Aims and Scope of JIDPS: Coping with the Challenges in Transdisciplinary Design}

\author{
Yong Zeng ${ }^{*}$ \\ Concordia Institute for Information Systems Engineering, Concordia University, Montreal, Canada
}

In my last editorial for Vol 18 No 4 of the journal (Zeng, 2014), the Aims and Scope of Journal of Integrated Design and Process Science (JIDPS) were summarized into a framework of three levels covering the research findings related to transdisciplinary notions of design and process: 1) understanding of design and process crossing boundaries of natural, human, and built environments, 2) principles, methods, and tools, and 3) applications of design and process science to engineering and social problems. In the last issue, three papers respectively addressing these issues were included.

A transdisciplinary design is a design that requires knowledge, expertise, experiences, and tools from different disciplines. There are many challenges in conducting a transdisciplinary design project. First, it is usually difficult for experts from different domains to communicate smoothly due to the different terminology and models that different domains use to describe the world. Second, due to the lack of an all-inclusive brain, the acquisition of relevant knowledge distributed in different domains can be a lengthy process. Third, the solution direction cannot be well defined before enough knowledge has been obtained while the necessary domain knowledge cannot be effectively and efficiently obtained before a solution direction has been well defined.

This issue will look into the concept of transdisciplinary design and associated methodologies and applications.

The first paper, titled "Environment-Based Design (EBD): a methodology for transdisciplinary design" by Zeng introduces a methodology to deal with transdisciplinary design problems. In the EBD methodology (Zeng, 2011), design is an activity that aims to change an existing environment to a desired one by creating a new artefact into the existing environment. As a result, design starts from the environment, serves the environment, and changes the environment. The transdisciplinary nature of a design can thus be taken into account through different kinds of environment and environment interactions across different disciplines. The EBD methodology includes three interdependent activities: environment analysis, conflict identification, and solution generation. Throughout the entire design process, any newly generated design solution will be viewed as an environment component for the succeeding design activities. The design process continues until no more undesired conflict exists in the environment. A transdisciplinary design problem can be decomposed through the partitioning of the environment implied in the problem description. Underlying the EBD methodology is a question strategy based on semantic analysis enabled by the Recursive Object Model (ROM). The questions generated throughout the design process will guide designers to collect information from different domains and to integrate the information into the present design context. In addition, this paper also proposes four criteria

\footnotetext{
*Corresponding author. Email: zeng@ciise.concordia.ca. Tel: (+1)514-8482424 ext. 5801.
} 
to evaluate the effectiveness of a design methodology: 1) to help designers jump out of the recursive loop between design problems, design knowledge, and design solutions, 2) to lead to both routine and creative design naturally, 3) to help a designer maintain his/her mental stress at an optimal level during the design process, and 4) to include naturally the conditions for the evolution of the design.

Among many existing design methodologies, EBD and TRIZ share one important foundation: conflict (contradiction) is the driving force behind design evolutions. TRIZ aims to support innovative design by identifying and resolving contradictions in a design (Altshuller, 1984). The identification of contradictions has been a bottle-neck for the efficient application of the TRIZ methodology (Savransky, 2000).

The second paper, titled "Systematic search for design contradictions in systems' architecture: toward a computer aided analysis" by Coatanéa et al., deals with contradictions in the TRIZ methodology. On the one hand, the increasing competition in the global market is imposing a great time pressure to the engineering design process, which pushes engineers to rush into known solutions before analysing properly the environment of a design and understanding the critical contradictions. On the other hand, the most important design decisions are made in early design phases, which will have a significant impact on later design tasks and activities. Among many ongoing efforts within different engineering communities to develop a model-based design approaches for early design stages, this present paper aims to develop a framework that can deal efficiently with the analysis of the early design concepts and architecture. The output from the proposed framework is an oriented graph with different types of classified variables, which can be processed to automatically identify the conflicts or contradictions present in the design concept architecture.

The third paper, titled "A fuzzy inventive problem-solving approach for product design: computeraided system using multi-agent and fuzzy TRIZ" by Chen, aims to automate the inventive problemsolving design process by developing a computer-aided system based on the concepts of multi-agent systems and fuzzy TRIZ. Their goal is to improve the efficiency of the invention process so that a corporate can consider both product innovation and environmental impact for customer's needs in a more effective manner. A case study of an actual notebook computer is used to compare the proposed method against other methods using Factor $\mathrm{X}$ indicator.

The fourth paper, titled "Depth sensors along the design and validation of lower limb prosthesis" by Regazzoni and Rizzi, falls into the category of interdisciplinary design. It is focused on different phases of lower limb prosthesis design and validation with the support of depth cameras. Two applications are reported in the paper. The first application consists in using depth sensors to perform a 3D scanning of still objects, including patients' anatomic districts. This application reconstructs the external geometry of an object. The second application uses depth cameras to measure positions of patient's body segments along the execution of a task.

Following the last and this issue, we will run a couple of more issues to further define the aims and scope of the journal. In addition to the topics covered in these two issues. Submissions are particularly welcome on research methodologies for transdisciplinary design. We also look forward to contributions from social sciences and arts.

\section{References}

Altshuller, G. S. (1984). Creativity as an Exact Science: The Theory of the Solutions of Inventive Problems (A. Williams, Trans.): Gordon and Breach Science Publishers.

Savransky, S. D. (2000). Engineering of Creativity: Introduction to TRIZ Methodology of Inventive Problem Solving: CRC Press.

Zeng, Y. (2011). Environment-Based Design (EBD). ASME 2011 International Design Engineering Technical Conferences and Computers and Information in Engineering Conference, 237-250

Zeng, Y. (2014). Aims and Scope of JIDPS: Understanding, Improving, and Practicing of Design. Journal of Integrated Design and Process Science, 18(4), 1-2. doi: 10.3233/jid-2014-0019 


\section{Author Biography}

Dr. Yong Zeng is a professor in the Concordia Institute for Information Systems Engineering at Concordia University, Montreal, Canada. Zeng's research interest is in understanding and improving design activities, especially creative design activities. In addition to developing a new design methodology Environment Based Design (EBD), he has been developing formal and experimental approaches to design research. He collaborates with aerospace companies, pharmaceutical companies, software development companies, and municipality for the applications of his research results. 